\title{
Avaliação Formativa usando Objetos de Aprendizagem SCORM
}

\author{
Renato Luís de Souza Dutra \\ Liane Margarida Rockenbach Tarouco \\ Liliana Passerino \\ Centro Interdisciplinar de Novas Tecnologias da Educação \\ Universidade Federal do Rio Grande do Sul
}

\section{Resumo:}

A Avaliação formativa tem ganhado cada vez mais adeptos nos últimos anos com a adoção de ciclos de aprendizagem e com a preocupação maior com o processo de ensino-aprendizagem. Na EAD, o processo de avaliação formativa tem sido bastante valorizado principalmente por permitir o constante feedback aos alunos e por permitir que professores e alunos regulem o processo de ensino-aprendizagem a distância.

O apoio ao processo de avaliação formativa nos diferentes Ambientes Virtuais de Aprendizagem (AVA), muitas vezes tem sido feito através de iniciativas que envolvem uso de sistemas multi-agentes e acompanhamento das atividades dos alunos. Entretanto, essas soluções possuem limitações como não a não portabilidade para diferentes AVA.

Nesse sentido, a utilização de Objetos de Aprendizagem SCORM, através de seu mecanismo de rastreamento é uma alternativa de apoio à avaliação formativa garantindo total portabilidade e interoperabilidade.

Palavras-chave: SCORM, Avaliação Formativa, Objetos de Aprendizagem, Avaliação da Aprendizagem

\section{Introdução}

A avaliação formativa apesar de ter sido inicialmente pensada por Scriven há mais de 30 anos (SCRIVEN, 1967), e ser discutida por diversos autores nas últimas décadas (BLOOM, HASTING \& MADAUS, 1983; PERRENOUD, 1999; CARDINET, 1986; HADJI, 2001) ainda é pouco aplicada. Somente nos últimos anos com a adoção de ciclos de aprendizagem e com a preocupação maior com o processo de ensino-aprendizagem, a avaliação com caráter mais formativo tem sido um pouco mais utilizada..

Em termos de EAD, a avaliação formativa tem se mostrado uma excelente alternativa, por propiciar aos professores um acompanhamento mais próximo de seus alunos e por permitir que professores e alunos regulem o processo de ensino-aprendizagem. 
Independente do processo avaliativo, o desenvolvimento de conteúdos digitais educacionais na forma de objetos de aprendizagem, tem sido uma solução crescente na EAD. Para tanto, padrões de interoperabilidade como o SCORM proporcionaram uma grande evolução em termos de reusabilidade, portabilidade e interoperabilidade, permitindo que o ambiente acadêmico possa facilmente intercambiar objetos de aprendizagem independente de plataforma de hardware e software. Dessa forma, o desenvolvimento de objetos de aprendizagem deveria levar em consideração o processo de avaliação formativa.

Esse artigo procura apresentar uma sugestão de utilização de elementos de dados do modelo SCORM, como forma de apoio para a avaliação formativa.

\section{Avaliação da Aprendizagem e Avaliação Formativa}

Especificamente na educação, a denominação "Avaliação da Aprendizagem” é atribuída a Ralph Tyler em 1930 (LUCKESI, 2006), considerado um dos principais precursores da avaliação da aprendizagem (LUCKESI, 2006; HOFFMAN, 1996; SAUL, 1998). Entre os diversos autores que sucederam Tyler, Michael Scriven, no final dos anos 60, desenvolveu uma série de idéias fundamentais na teoria de avaliação educacional. Sua principal contribuição, no entanto, foi diferenciar o papel formativo e somativo da avaliação, conceitos que influenciaram de maneira singular as pesquisas em avaliação.

Complementando os trabalhos de Tyler, Bloom et al. (1972) criaram a taxonomia de objetivos educacionais, identificando e subdividindo os objetivos educacionais em três domínios: o cognitivo, o afetivo e o psicomotor. Em estudos posteriores e ampliando as contribuições de Scriven, Bloom et al. (1983) classificaram as funções da avaliação em diagnóstica, somativa e formativa. Para os autores, a avaliação diagnóstica envolve a determinação do valor no sentido de diagnosticar seu domínio dos objetivos previstos e necessários para se iniciar uma atividade de ensino. A avaliação formativa ocorre durante o processo de ensino-aprendizagem, fornecendo feedback a professores e alunos acerca do que o aluno aprendeu, do que ele necessita aprender, de quais são suas necessidades individuais e quais aspectos da instrução precisam ser modificados, com o intuito de individualizar o atendimento ao aluno e solucionar as falhas na aprendizagem. Já a avaliação somativa envolve a descrição, a classificação e a determinação do valor de algum aspecto do aluno, no sentido de auxiliar o julgamento para classificar os alunos ao final de uma unidade, semestre ou ano, expressos em graus ou conceitos.

\subsection{Avaliação Formativa}

O termo avaliação formativa foi proposto por Scriven pela primeira vez em $1963 \mathrm{em} \mathrm{seu}$ artigo "Evaluation for Course Improvement", definindo-o como um dos papéis da avaliação como contraponto à avaliação somativa (SCRIVEN, 1967). Outros autores também ratificam Scriven como o criador do termo 'avaliação formativa', bem como sua 
essência principal (BLOOM et al., 1983; PERRENOUD, 1999; HADJI, 2001; LUCKESI, 2006).

Scriven (1967) se referia à avaliação formativa remetendo aos estudos de Cronbach em que este afirmava que a avaliação utilizada para melhorar o curso durante seu andamento contribuía mais para a melhoria da educação do que uma aplicada após o produto já estar no mercado (CRONBACH, 1966 apud SCRIVEN, 1967). Scriven (1967) utilizou-se desta idéia propondo, assim, a avaliação formativa como um instrumento no qual o professor estaria altamente engajado na melhoria de seu material (posteriormente esta concepção mudou para pensar também a metodologia de ensino, as necessidades de seus alunos, como veremos mais adiante). Desta forma, aplicando testes e validando o andamento das aprendizagens durante o desenrolar do curso poderia colher subsídios para sua melhoria. Bloom e seus colaboradores (1983), em seu livro Manual da Avaliação Formativa e Somativa, ampliam a visão de Scriven para o processo de ensino/aprendizagem, propõem alguns instrumentos para avaliação formativa e assim definem esse tipo de avaliação:

A avaliação formativa busca basicamente identificar insuficiências principais em aprendizagens iniciais, necessárias à realização de outras aprendizagens. Providencia elementos para, de maneira direta, orientar a organização do ensino-aprendizagem em etapas posteriores de aprendizagem corretiva ou terapêutica. Neste sentido, deve ocorrer freqüentemente durante o ensino (BLOOM et al., 1983).

Apesar de sua primeira proposição ter ocorrido há quase 50 anos, sua aplicação tem sido mais significativa nas últimas décadas, a partir das experiências de ciclos de aprendizagem, na preocupação de respeitar as diferenças individuais dos alunos e de regular melhor o processo de ensino-aprendizagem durante o processo (GATHER THURLER, 2001; PERRENOUD, 2003). Mesmo assim, ainda hoje a avaliação formativa é pouco utilizada. Uma causa apontada por Perrenoud (1999) é que muitas vezes os professores pensam que para aplicar a avaliação formativa é necessária a utilização de novas pedagogias, entretanto a avaliação formativa pode ser aplicada a qualquer tipo de pedagogia.

Apesar das diferenças nas concepções de avaliação formativa, a partir de Bloom elas têm em comum a mesma essência. Todos os autores sugerem que a avaliação formativa é realizada durante o processo de ensino/aprendizagem, que ela é contínua, que ela não deve ser característica classificatória ou certificadora e que se baseia fortemente no feedback tanto para o professor como para o aluno (BLOOM et al.,1983; ZABALA, 1998; PERRENOUD, 1999; HADJI, 2001). Pode-se perceber que as primeiras propostas de avaliação formativa estavam mais focadas no aprimoramento dos conteúdos e do programa (SCRIVEN, 1967), com ampliação para o processo de ensino e aprendizagem (BLOOM et al.,1983). Já as propostas mais recentes passaram a se preocupar mais com o processo de regulação na tentativa de adequar o processo de ensino/aprendizagem de acordo com as características individuais de cada aluno. Nesse 
sentido, Cardinet (1986), Perrenoud (1986;1999) e Hadji (2001) têm feito contribuições importantes, com bastante preocupação no processo de regulação das aprendizagens a partir da avaliação formativa.

Hadji (2001) acrescenta dizendo que a avaliação formativa apresenta três características principais: ela é informativa, pois informa os dois atores do processo de ensinoaprendizagem - ao professor, que será informado dos efeitos reais de suas ações, podendo regular sua ação pedagógica, e ao aprendiz, que terá oportunidade de tomar consciência de suas dificuldades e, possivelmente, reconhecer e corrigir seus próprios erros; ela é reguladora, pois permite ao professor e ao aluno corrigir suas ações, modificando-as, se necessário, a fim de obter melhores resultados.

Através da avaliação formativa, o professor avalia os efeitos de sua ação, verificando a compatibilidade com os objetivos propostos, modificando sua ação para melhor atingir seus objetivos (KENSKI, 2007; PERRENOUD, 1999). Essa avaliação, portanto, ajuda o professor a observar de forma mais sistemática seus alunos, compreendendo-os melhor para, desse modo, conseguir ajustar de maneira mais organizada e individualizada as atividades didáticas que propõe, buscando sempre otimizar as aprendizagens dos alunos (PERRENOUD, 1999).

Pode-se dizer então que a avaliação formativa não vem para excluir os outros modelos de avaliação. A avaliação formativa induz à reflexão, tanto do aluno como do professor, e ajuda-os de forma dialógica a aperfeiçoar e ajustar suas ações e atividades.

\section{Avaliação Formativa na EAD}

Antes mesmo da popularização da Internet e surgimento da Web, Santarosa (1982) já observava que a avaliação formativa apoiada por computador poderia conduzir a um melhor desempenho do aluno. No seu entendimento, a grande capacidade de armazenamento de dados e a possibilidade de feedback individual a uma maior quantidade de alunos já sugeriam fortemente a utilização dos computadores para apoiar a avaliação formativa.

Já quando se observam as particularidades da $\mathrm{EAD}$, em que existe uma grande diversidade no grupo de aprendizes, é ainda mais interessante realizar avaliações durante o processo de ensino-aprendizagem, sem atribuições de notas e disponibilizando feedback detalhado e constante aos alunos (SIMOLSON et al. 2000), características estas proporcionadas pela avaliação formativa. $\mathrm{Na}$ verdade, a avaliação formativa apresenta-se como o instrumento mais apropriado para acompanhar a evolução e a performance do aluno em um curso a distância (ZAINA, 2002). Sendo assim, qualquer dificuldade encontrada pelos aprendizes pode ser orientada antes que se torne um grande obstáculo à aprendizagem (SIMOLSON et al., 2000).

O que se observa é que a avaliação formativa tem se mostrado de vital importância para apoiar a percepção do comportamento dos aprendizes a distância, pois proporciona uma 
orientação mais adequada à atividade em andamento, em um contexto em que o professor não tem contato presencial com o aluno (OTSUKA, 2006).

Nesse sentido, a utilização de AVAs na avaliação formativa oferece uma riqueza de informações registradas no ambiente, cabendo ao professor transformar esse conjunto de informações em subsídios para uma avaliação (KENSKI et al., 2006). Entretanto, é importante haver um suporte adequado ao processo de avaliação formativa on-line, pois esse modelo de avaliação pode gerar uma grande sobrecarga para os professores (OTSUKA, 2006; PRATA, 2003). Essa sobrecarga normalmente se dá pela necessidade de análise de informações sobre a participação do aluno, dispersas no AVA.

Diversos estudos e trabalhos já foram realizados no sentido de apoiar a avaliação formativa na EAD. Esses estudos incluem a utilização dos AVA, de seus mecanismos de rastreamento e de Sistemas Multi-Agentes. Entre esses trabalhos poderíamos citar Weirich e seus colaboradores (2007), Otsuka (2006), Rodrigues (2002), Prata (2002), Pimentel e seus colaboradores (2007), entre outros.

Em todos esses trabalhos observa-se que um mecanismo importante para o processo de avaliação é o rastreamento das atividades e ações dos alunos dentro do AVA. Outra informação utilizada é a oriunda do registro de respostas dos exercícios que foram realizados dentro do próprio AVA. Entretanto, o que se verifica é que nenhuma das soluções pode ser facilmente portada para outros AVAs, por terem sido desenvolvidas especificamente para um determinado AVA. Nesse sentido, é necessária a busca de soluções permitam uma fácil portabilidade e reusabilidade dos recursos de avaliação, tendo em vista a grande diversidade de AVAs utilizados por instituições de ensino no Brasil e no mundo. Uma alternativa é a utilização de Objetos de Aprendizagem SCORM como mecanismo de avaliação formativa.

\section{SCORM}

SCORM é um acrônimo de Sharable Content Object Reference Model ou Modelo de Referência para Objetos de Aprendizagem Compartilháveis. O SCORM não é um padrão, e sim um modelo de referência, o que significa que ele é um conjunto unificado de recomendações para conteúdos e serviços de e-learning. Este modelo de referência sugere quais serviços são necessários para cursos e treinamentos on-line via Web, como esses serviços podem ser 'empacotados', quais padrões existentes devem ser aplicados, e também a maneira como esses padrões devem ser utilizados (ADL, 2004).

O modelo SCORM é formado por um conjunto de especificações publicados na forma de livros. Para o SCORM 1.2, a ADL publicou as especificações em três livros: Visão Geral (The SCORM Overview), Modelo de Agregação de Conteúdo (The SCORM Content Aggregation Model) e Ambiente de Execução (The SCORM Runtime Environment). Já para o SCORM 2004, a ADL corrigiu alguns defeitos e acrescentou o livro Seqüenciamento e Navegação (The SCORM Sequencing \& Navigation). A figura 1 
ilustra a organização do SCORM 2004 como um conjunto de especificações de outras organizações contidas ou referenciadas no modelo.

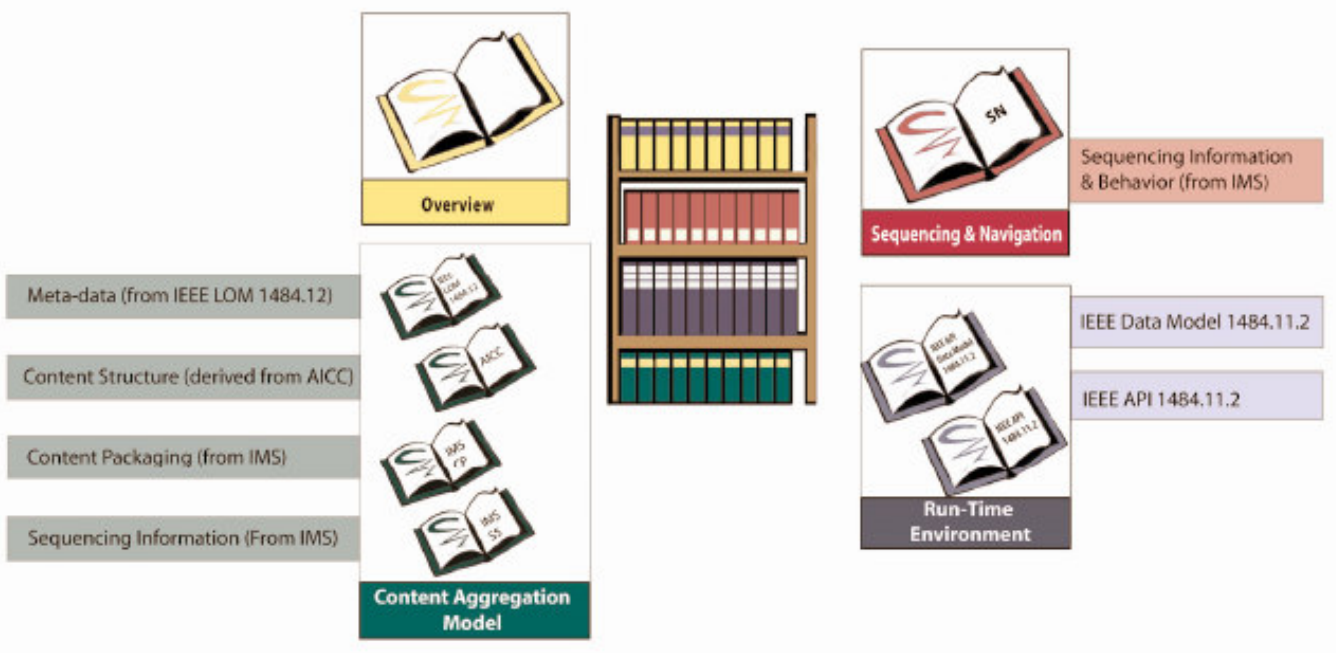

Figura 1.1 - SCORM como conjunto de especificações

O SCORM preocupa-se com o conteúdo, em como ele é estruturado e como será sua navegação. Entretanto, sua principal característica é rastrear as ações do aluno dentro do AVA, no que diz respeito à interação do conteúdo propriamente dito. Essas informações estão descritas no livro 'Ambiente de Execução', que define um Mecanismo de Execução comum para comunicação entre o AVA e os objetos de aprendizagem SCORM (SCO), bem como um Modelo de Dados comum para apoiar essa comunicacao e rastrear as atividades do aluno.

\subsection{Utilização do SCORM na Avaliação Formativa}

O SCORM, em seu Ambiente de Execução, estipula um modelo de dados que contém elementos que podem ser intercambiados entre o AVA e os objetos de aprendizagem, nesse caso os objetos de aprendizagem SCORM (SCO). Alguns desses elementos são coletados automaticamente pela simples inicialização e finalização do objeto de aprendizagem SCORM (SCO), outros precisam ser programados para tal fim dentro dos SCO através de chamadas padronizadas na linguagem JavaScript. Esses elementos podem se registrados de forma correta e observados através dos relatórios de rastreamento dos AVAs, podem ser utilizados para a avaliação formativa. O quadro 4.1 demonstra quais elementos do SCORM podem ser utilizados para esse fim.

\section{\begin{tabular}{|l|l|l}
\hline Elemento do & Notação do elemento & Descrição \\
\hline
\end{tabular}}




\begin{tabular}{|c|c|c|}
\hline modelo de dados & & \\
\hline $\begin{array}{l}\text { Comments From } \\
\text { Learner }\end{array}$ & cmi.comments_from_learner & Contém comentários e textos do aprendiz sobre o SCO. \\
\hline $\begin{array}{l}\text { Comments From } \\
\text { LMS }\end{array}$ & cmi.comments_from_lms & $\begin{array}{l}\text { Contém comentários e anotações do SCO com o } \\
\text { objetivo de serem mostradas para os aprendizes. }\end{array}$ \\
\hline Completion Status & cmi.completion_status & Indica quando o aprendiz terminou o SCO. \\
\hline Interactions & cmi.interactions & $\begin{array}{l}\text { Define informações pertinentes às interações (testes, } \\
\text { exercícios, etc.) com o propósito de medição ou } \\
\text { avaliação. }\end{array}$ \\
\hline Learner Name & cmi.learner_name & $\begin{array}{l}\text { Representa o nome do aprendiz que está executando o } \\
\text { SCO. }\end{array}$ \\
\hline Objectives & cmi.objectives & $\begin{array}{l}\text { Especifica os objetivos de aprendizagem ou performance } \\
\text { associados com cada SCO. }\end{array}$ \\
\hline Progress Measure & cmi.progress_measure & $\begin{array}{l}\text { Mede o progresso que o aluno está tendo navegando pelo } \\
\text { SCO. }\end{array}$ \\
\hline $\begin{array}{l}\text { Scaled Passing } \\
\text { Score }\end{array}$ & cmi.scaled_passing_score & Identifica a pontuação scaled para um SCO. \\
\hline Score & cmi.score & Identifica a pontuação do aluno para um SCO. \\
\hline Session Time & cmi.session_time & $\begin{array}{l}\text { Identifica o tempo que o aluno gastou na sessão corrente } \\
\text { do SCO. }\end{array}$ \\
\hline Success Status & cmi.success_status & Indica quando o aluno finalizou com sucesso o SCO. \\
\hline Total Time & cmi.total_time & $\begin{array}{l}\text { Identifica o tempo total acumulado de todas as sessões } \\
\text { na tentativa corrente do aluno. }\end{array}$ \\
\hline
\end{tabular}

Quadro 1.1 - Conjunto de Elementos importantes para a Avaliação Formativa

Para cada um dos elementos mostrados no quadro 6.1 existe um conjunto de subelementos (também referenciados como elementos nos livros SCORM) que efetivamente armazenam todos os dados do elemento, e que são intercambiados pelo AVA e SCOs através de comandos de recuperar (get) e registrar (set).

Os comentários do aluno (cmi.comments_from_learner) podem ser utilizados para registro das impressões sobre a experiência de aprendizagem no SCO, para registrar anotações de um diário de bordo e para armazenar respostas a questões dissertativas, entre outras coisas. Já os comentários do AVA (cmi.comments_from_lms) contêm informações que devem ser vistas pelos alunos quando estes estiverem navegando nos SCOs, desde que o SCO contenha tais elementos registrados. O SCORM não define o mecanismo pelo qual esses elementos serão inicializados, mas define que estes devem ser registrados somente a partir do AVA, cabendo ao SCO o direito de recuperar e mostrar essas informações (ADL, 2006).

O conjunto de elementos das interações (cmi.interactions) é uma forma de registrar as respostas dos alunos em exercícios. As interações são respostas para exercícios ou 
atividades individuais que se queiram registrar em um SCO. Para o AVA, a única obrigação é registrar esses dados quando solicitados, não tendo que proceder com nenhum comportamento específico. São excelentes instrumentos para a avaliação formativa por permitirem o registro da resposta exata do aluno em tais exercícios, possibilitando a análise posterior do professor. Com os diversos tipos de interação permitidos, é possível inserir tais interações para avaliar o nível de dificuldade dos alunos.

Outro conjunto de elementos úteis do SCORM são os objetivos educacionais (cmi.objectives), em que para cada SCO podem ser identificados um ou mais objetivos. Os objetivos são tratados como agrupamentos do conjunto de elementos de dados dos objetivos para um determinado $\mathrm{SCO}$, os quais podem ser usados para rastrear a aprendizagem do aluno.

Já o elemento do modelo de dados intitulado de 'Score' (cmi.score) é utilizado para registrar a pontuação ou nota do aluno no SCO. Muitas vezes, no fim de cada SCO, uma avaliação objetiva pode ser introduzida para avaliar o desempenho do aluno no SCO, registrando sua pontuação. Apesar de seu aparente enfoque somativo, a pontuação pode ser mais um observável no processo de avaliação formativa, desde que utilizado com outros exercícios e analisado no conjunto de elementos registrados para um SCO.

O tempo de Sessão (cmi.session_time) é um dos elementos mais utilizados nos relatórios de atividades SCORM e demonstra o total de tempo que o aluno gastou na última sessão para determinado SCO, sendo que o tempo total (cmi.total_time) é a soma de todos os tempos de sessão registrados para o SCO. Esses elementos são utilizados para acompanhar o tempo gasto pelos alunos em todas as suas sessões para uma determinada tentativa (ADL, 2006). Através dele, o avaliador pode verificar se o aluno está levando mais tempo para navegar por um determinado SCO do que outros alunos, o que pode indicar possíveis dificuldades do aluno naquela parte do conteúdo.

A medida de progresso (cmi.progress_measure) é o elemento utilizado para aferir o progresso do aluno na execução do SCO. Pode ser definido como 'not attempted' para quando ainda não foi executado, 'completed' para quando foi executado até o final ou 'incomplete' quando foi executado sem chegar ao final. O mecanismo de definição do valor desse elemento é determinado pelo desenvolvedor e estará implícito no algoritmo do SCO. Através desse elemento, é possível que o avaliador verifique se o aluno conseguiu chegar ou não ao final do SCO. Em conjunto com os elementos tempo total e registro do número de tentativas, o avaliador pode inferir se o aluno está tendo dificuldades nos conteúdos estudados.

O último elemento recomendado para o apóia à avaliação formativa é o status do sucesso (cmi.success_status). Ele indica quando o aluno atingiu com êxito o final do SCO. O SCORM não define como esse sucesso deve ser determinado, mas o SCO pode indicar esse status baseando-se no percentual das interações realizadas, objetivos alcançados, pontuação atingida em uma interação, etc. Como o critério de sucesso é 
determinado pelo desenvolvedor do SCO, ele pode ser utilizado de acordo com as diretrizes do avaliador para facilitar o acompanhamento do aluno na avaliação formativa (ADL, 2006).

Todos esses elementos para serem corretamente registrados, demandam a intervenção do desenvolvedor do objeto de aprendizagem incluindo comandos que invoquem seu correto registro, sendo muitas vezes necessária a readequação e a adição de comandos em objetos de aprendizagem existentes. Além disso, é necessário o empacotamento desses objetos de aprendizagem já modificados em unidades de aprendizagem SCORM, por meio de ferramentas de empacotamento, preferencialmente de software livre.

Atualmente, existe no mercado uma ampla gama de ferramentas de autoria, utilizadas para produzir conteúdo educacional digital para Educação a Distância. Ferramentas de criação de páginas, além do Flash, situam-se no topo da lista das mais utilizadas. Nesse contexto, há softwares comerciais e livres que oferecem suporte para transformar páginas HTML com interatividade em pacotes compatíveis com o modelo SCORM, AICC e outros padrões de mercado. É cada vez mais usual uso de ferramentas de autoria criadas especificamente para o desenvolvimento de conteúdos educacionais digitais, que além de ajudarem no desenvolvimento desses conteúdos, encapsulam e empacotam diretamente em SCORM. Entre as ferramentas para esse fim de código aberto ou simplesmente o uso livre destacam-se o Hot Potatoes, o Xerte, e o CourseLab. Além disso, por meio de ferramentas tais como Click2Learn SCORM Tools, Xerte, Reload Editor, eXe Learning, IOPackager (IOP \& MEDINA, 2007), é possível seqüenciar e/ou empacotar conteúdos desenvolvidos anteriormente para Web em conteúdos SCORM.

\section{Conclusões}

O processo de avaliação formativa na EAD é cada vez mais valorizado principalmente por permitir o constante feedback aos alunos e por permitir que professores e alunos regulem o processo de ensino-aprendizagem a distância.

O apoio ao processo de avaliação formativa nos diferentes Ambientes Virtuais de Aprendizagem (AVA) tem sido feito através de iniciativas que envolvem uso de sistemas multi-agentes e acompanhamento das atividades dos alunos. Entretanto, essas soluções especificas não podem ser portadas para diferentes AVA, dificultando sua adoção em maior escala.

O uso de objetos de aprendizagem SCORM de maneira a apoiar o processo de avaliação formativa pode ser uma alternativa interessante por ser uma solução que garante total portabilidade e interoperabilidade. Para isso, o SCORM possui elementos de dados que são registrados pelos AVA e que se usados corretamente, podem ser utilizados na avaliação formativa. Entre esses elementos destacamos, o numero de tentativas, o tempo de acesso, o registro das respostas nos exercícios, a pontuação e o registro de comentário do aluno. 
O desenvolvimento de objetos de aprendizagem SCORM (SCO) levando em consideração o registro de tais elementos de forma a serem utilizados na avaliação formativa é uma alternativa ainda pouco explorada, mas que se utilizada de forma eficaz, é uma forma mais flexível de utilização de recursos para apoiar a avaliação formativa sem se prender a um AVA ou mecanismo de avaliação especifico.

\section{Referências:}

ADL. Advanced Distributed Learning . SCORM 2004 3rd edition. Version 1.0. 2006. Disponível em: <http://www.adlnet.org>. Acesso em: 15 fev. 2007.

BLOOM, B. S.; KRATHWOHL, D. R.; MASIA, B. B. Taxionomia de objetivos educacionais. Porto Alegre : Globo, 1972.

BLOOM, B. S.; HASTINGS, J. T.; MADAUS, G. F. Manual de Avaliação Formativa e Somativa do Aprendizado Escolar. S. Paulo: Livraria Pioneira Editora, 1983.

CARDINET, J. Linhas de desenvolvimento dos trabalhos actuais sobre a avaliação formativa. In: ALLAL, L.; CARDINET, J.; PERRENOUD, P. A Avaliação Formativa num Ensino Diferenciado. Coimbra: Livraria Almedina, 1986.

GATHER THURLER, Mônica. Quais as competências para operar em ciclos de aprendizagem plurianuais ? IN: Pátio. Ano V, nº 17, mai/jul. 2001, pg 17-21.

HOFFMANN, J. Avaliação: Mito e Desafio - Uma perspectiva construtivista. Porto Alegre: Mediação, 2001.

IOP, R. D.; MEDINA, R. D. IOPackager - Desenvolvimento de uma ferramenta automática conversora de objetos educacionais em pacotes de conteúdo SCORM 2004 3rd edition. RENOTE - Revista Novas Tecnologias na Educação: IX Ciclo de Palestras sobre Novas Tecnologias na Educação, Porto Alegre, v. 5 n. 1, 2007.

KENSKI, V. M.; OLIVEIRA, G. P.; CLEMENTINO, A. Avaliação em movimento: estratégias formativas em cursos online. In: SILVA, M.; SANTOS, E. (Org.). Avaliação da Aprendizagem em Educação Online. São Paulo: Loyola, 2006.

LUCKESI, Cipriano C. Avaliação da Aprendizagem Escolar: estudos e proposições. São Paulo: Cortez, 2006.

OTSUKA, J. L. Modelo de Suporte à Avaliação Formativa Baseado em Sistemas Multiagentes para Ambientes de EaD. Tese de doutorado. Instituto de Computação-UNICAMP, 2006.

PERRENOUD, P. Das diferenças culturais às desigualdades: a avaliação e a norma num ensino indiferenciado. In: ALLAL, L.; CARDINET, J.; PERRENOUD, P. A Avaliação Formativa num Ensino Diferenciado. Coimbra: Livraria Almedina, 1986. 
PERRENOUD, P. Avaliação: Da Excelência à Regulação das Aprendizagens entre duas lógicas. Porto Alegre: Artmed, 1999.

PERRENOUD, Philipe. Os Ciclos de Aprendizagem: Um Caminho para Combater o Fracasso Escolar. Porto Alegre: Artmed, 2003.

PIMENTEL, E. P.; ALVES, A. S.; OLIVEIRA, D. M. IKEHARA; BOTTARO, P. A.; LOPES, R. Avaliações Adaptativas baseadas no Nível de Aquisição de Conhecimento do Aprendiz. In: Anais do XVIII Simpósio Brasileiro de Informática na Educação, SBIE 2007. São Paulo - SP Universidade Presbiteriana Mackenzie e USP; SBC, Porto Alegre, 2007.

PRATA, D. N. Estratégias para o Desenvolvimento de um Framework de Avaliação na Aprendizagem a Distância. In: Anais do XVI Simpósio Brasileiro de Informática na Educação.2003.

RODRIGUES, Alessandra Pereira. E-Avalia - Um agente para a avaliação de EnsinoAprendizagem em Educação a Distância. Dissertação de Mestrado, Programa de Pós-Graduação em Computação, Instituto de Informática-UFRGS, 2002.

SANTAROSA, L. M. C. O Computador na Avaliação Formativa: Efeitos Interativos com a Ansiedade e Atitude sobre o Comportamento dos Alunos. Porto Alegre: UFRGS, 1982.

SAUL, Ana Maria. Avaliação Emancipatória: desafio à teoria e à pratica de avaliação e reformulação de currículo. São Paulo: Cortez: Autores Associados, 1988.

SCRIVEN, M The Methodology of Evaluation. In: TYLER, R.; GAGNE, R.; SCRIVEN, M. Perspectives of Curriculum Evaluation. Washington, D.C: American Educational Research Association, 1967.

SIMONSON, M.; SMALDINO, S.; ALBRIGHT, M.; ZVACEK, S. Teaching and Learning at a Distance: Foundations of Distance Education. Upper Saddle River: Prentice-Hall, 2000.

ZAINA, Luciana Aparecida Martinez. Acompanhamento do aprendizado do aluno em cursos à distância através da Web: metodologias e ferramenta. Dissertação de Mestrado, Departamento de Engenharia da Computação, Escola Politécnica da Universidade de São Paulo, São Paulo, 2002. 